\title{
Gut Microbiota and Pathophysiology of Depressive Disorder
}

\author{
Hiroshi Kunugi \\ Department of Psychiatry, Teikyo University School of Medicine, Tokyo, Japan
}

\section{Keywords}

Gut microbiota · Pathophysiology · Probiotics · Major depressive disorder

\begin{abstract}
Background: Accumulating evidence has suggested that the bi-directional communication pathway, the microbiota-gutbrain axis, plays an important role in the pathophysiology of many neuropsychiatric diseases including major depressive disorder (MDD). This review outlines current evidence and promising findings related to the pathophysiology and treatment of MDD. Summary: There are at least 4 key biological molecules/systems underlying the pathophysiology of MDD: central dopamine, stress responses by the hypothalamic-pituitary-adrenal axis and autonomic nervous system, inflammation, and brain-derived neurotrophic factor. Animal experiments in several depression models have clearly indicated that gut microbiota is closely related to these molecules/ systems and administration of probiotics and prebitotics may have beneficial effects on them. Although the results of microbiota profile of MDD patients varied from a study to another, multiple studies reported that bacteria which produce short-chain fatty acids such as butyrate and those protective against metabolic diseases (e.g., Bacteroidetes) were reduced. Clinical trials of probiotics have emerged, and the ma-
\end{abstract}

karger@karger.com www.karger.com/anm

Karger $\stackrel{\text { ' }}{5}$ (c) 2021 The Author(s).

Published by S. Karger AG, Basel

This is an Open Access article licensed under the Creative Common Attribution-NonCommercial-4.0 International License (CC BY-NC) (http://www.karger.com/Services/OpenAccessLicense), applicable to the online version of the article only. Usage and distribution for commercial purposes requires written permission. jority of the studies have reported beneficial effects on depression symptoms and related biological markers. Key Messages: The accumulating evidence suggests that research on the microbiota-gut-brain axis in major depressive disorder (MDD) is promising to elucidate the pathophysiology and to develop novel treatment of MDD, although there is still a long distance yet to reach the goals.

(C) 2021 The Author(s)

Published by S. Karger AG, Basel

\section{Introduction}

Depressive disorder is a common, debilitating psychiatric disease characterized by depressed mood, anhedonia, altered appetite, sleep disturbance, psychomotor retardation/agitation, fatigue, guilt feeling, loss of concentration, and suicidal ideation with the global point prevalence estimated at $4.4 \%$ worldwide [1]. Large-scale clinical trials have revealed that a substantial proportion of patients with major depressive disorder (MDD) fail to respond to, or achieve remission with, the current firstline antidepressants [2]. Also, many patients with MDD show poor adherence to the current antidepressants due to their adverse effects, which requires new treatment strategies with less adversity. However, the pathophysiology of MDD remains elusive, and there is no established 
biochemical marker used in the daily clinical setting. This situation may result in part from the heterogeneity of MDD which might include heterogeneous subgroups with different biological mechanisms or combination of them. There are at least 4 promising biological systems/ markers to potentially subtype MDD, that is, the dopamine system, stress response by the hypothalamic-pituitary-adrenal (HPA) axis and autonomic nervous system (ANS), chronic inflammatory markers, and dysfunction of brain-derived neurotrophic factor (BDNF) [3-5]. Growing evidence has suggested that altered gut microbiota may be involved in the pathophysiology of depressive disorder [6, 7]. In this review, related evidence is outlined in relation to the pathophysiology of MDD.

\section{Pathophysiology of Depressive Disorder}

\section{Dopamine System}

The monoamine hypothesis might be the best-known etiological hypothesis for MDD. Any classes of antidepressants exert their effects through increasing monoamine neurotransmitters (serotonin, noradrenaline, and/ or dopamine) in the synaptic cleft. Given the findings from in vitro studies that first-line antidepressants such as selective serotonin reuptake inhibitors (SSRIs) and serotonin noradrenaline reuptake inhibitors inhibit the reuptake of serotonin and noradrenaline, one might suspect that these 2 neurotransmitters play more important roles than dopamine. However, the recent evidence from the cerebrospinal fluid (CSF) suggests its opposite direction. Among monoamine metabolites, homovanillic acid (HVA, a primary dopamine metabolite) but not 5-hydroxyindoleacetic acid (a primary serotonine metabolite), or 3-methoxy-4-hydroxyphenylglycol (MHPG: a primary noradrenaline metabolite), was shown to be significantly reduced in the CSF of MDD patients [8-10]. In line with this, in vivo animal studies using microdialysis have demonstrated that many of the SSRIs increase extracellular dopamine as well as serotonin in the prefrontal cortex, despite the fact that SSRIs do not inhibit the reuptake of dopamine in vitro. The increase in dopamine could be central to the antidepressant effects [11]. Indeed, at least a portion of MDD is responsive to dopamine agonists $[12,13]$, and such drugs increase BDNF levels in the cerebral cortex and hippocampus in animal studies $[14,15]$.

\section{Altered Stress Response}

MDD is often induced by chronic stress; therefore, abnormal stress response in the HPA axis is one of the most extensively studied biological markers for depression [16]. Initially, typical MDD subtypes such as melancholic and psychotic types were found to have enhanced HPA activity due to inadequate negative feedback of the HPA axis detected by hormonal challenge tests such as the dexamethasone (DEX) suppression test and DEX/corticotropin-releasing hormone $(\mathrm{CRH})$ test. In line with this, we also reported enhanced cortisol responses in the DEX/ $\mathrm{CRH}$ test in Japanese MDD inpatients before treatment and its recovery after treatment $[17,18]$. However, subsequent studies have revealed that depressed patients as a whole showed similar [19], or even attenuated [20], cortisol responses as compared to healthy controls in the DEX/CRH test. In these studies, patients had clinical characteristics such as outpatients, chronic cases, depressive patients with psychiatric comorbidity, or long-term sick-leave patients. These inconsistent findings are related to the heterogeneity of the illness. Indeed, atypical depression and other stress-related conditions such as posttraumatic stress disorder, chronic fatigue syndrome, and fibromyalgia are associated with attenuated, rather than enhanced, cortisol responses in the HPA axis [21-23]. It is also well-known that somatic diseases such as Addison's disease and ACTH deficiency, both of which show inadequate cortisol secretion, present psychiatric symptoms similar to MDD. These observations clearly lead to the possibility that HPA axis activity can be a subtyping marker of MDD.

The ANS is another important player in stress response. Imbalance in sympathetic and parasympathetic nervous systems has been suggested in patients with MDD which could be monitored by the heart rate variability [24]. Excessive and attenuated activations of sympathetic and parasympathetic nervous systems, respectively, have been suggested to be underlying in the pathophysiology of MDD. In line, there is some evidence that vagus nerve stimulation is effective in treatment-resistant depression [25], which is closely related to the following gut microbiota described.

\section{Inflammation}

Accumulating evidence suggests that a portion of MDD has been reformulated as a chronic inflammatory disease similar to diabetes and coronary heart disease in which pro-inflammatory cytokines such as interleukin (IL)-6), IL-1 $\beta$ and tumor necrosis factor- $\alpha$ contribute to the disease process [26]. Elevation in peripheral pro-inflammatory cytokines leads to neural inflammation; indeed, we reported increased IL- 6 levels in the CSF of patients with MDD [27], which was subsequently confirmed
12

Ann Nutr Metab 2021;77(suppl 2):11-20 DOI: $10.1159 / 000518274$
Kunugi 
by a meta-analysis [28]. An important mechanism of cytokine-induced depression is the activation of the tryptophan-kynurenine pathway [29]. In line, we provided evidence supporting the elevated activation of tryptophankynurenine pathway by using ${ }^{13} \mathrm{C}$-tryptophan breath test in patients with MDD [30]. Further, we showed decreased plasma tryptophan levels in patients with $\mathrm{MDD}[31,32]$, which is likely to be due, at least in part, to the enhanced tryptophan-kynurenine pathway. In the brain, kynurenine is converted to quinolinic acid or 3-hydroxykynurenine in microglia, which has neurotoxic effect through the property as an N-methyl-D-aspartate receptor (NMDAR) agonist and is thought to be involved in the pathogenesis of depression.

\section{Brain-Derived Neurotrophic Factor}

Several lines of evidence from postmortem studies, animal studies, blood levels, and genetic studies have suggested that BDNF is involved in the pathogenesis of depression and in the mechanism of action of biological treatments of depression [33]. There is convincing evidence that stress reduces the expression of BDNF and that antidepressant treatments increase it $[33,34]$. Moreover, glucocorticoid receptor interacts with the specific receptor of BDNF, tropomyosin receptor kinase B (TrkB), and excessive glucocorticoid interferes with BDNF signaling $[3,35,36]$. Altered BDNF function is involved in the structural changes and possibly impaired neurogenesis in the brain of depressed patients. Although BDNF levels seem to be undetectably low in the human CSF, we found reduced $\mathrm{BDNF}$ propeptide, which is produced in the processing of pro-BDNF to mature BDNF, in patients with MDD compared with healthy controls [37].

\section{Animal Studies Linking Gut Microbiota and Depressive-Like Behavior}

A number of studies on animal models of depressive disorder have thus far been conducted. Here, some of the cornerstone studies are introduced. Sudo et al. [38] provided evidence suggesting the importance of commensal microbiota in the development of healthy stress response in the HPA axis. They found that plasma ACTH and corticosterone elevation in response to restraint stress was substantially higher in germ-free (GF) mice than in specific pathogen-free (SPF) mice. Moreover, GF mice exhibited reduced BDNF expression levels in the cortex and hippocampus relative to SPF mice. The exaggerated HPA stress response by GF mice was reversed by reconstitution

Gut Microbiota and Depression with Bifidobacterium (B.) infantis. Further, the enhanced HPA response of GF mice was partly corrected by reconstitution with SPF feces at an early stage but not by any reconstitution exerted at a later stage, indicating that exposure to microbes at an early developmental stage is required for the healthy development of the HPA system. Crumeyrolle-Arias et al. [39] reported heightened anxiety-like behaviors and elevated CRH mRNA expression in the hypothalamus and reduced glucocorticoid receptor mRNA expression in the hippocampus in GF adult rats compared to SPF rats. They observed a significant decrease in HVA-to-dopamine turnover ratio (HVA/dopamine) in the prefrontal cortex, hippocampus, and striatum of GF adult rats compared to SPF rats, suggesting that gut microbes are important in the development of dopaminergic neurochemistry. Also, Liu et al. [40] demonstrated that administration of Lactobacillus (L.) plantarum PS128 to GF mice decreased anxiety-like behaviors and that these changes were accompanied by an increase in DA and HVA, as well as an increase in serotonin in the striatum.

In a rat maternal separation (MS) model of depression, altered colonic functions such as increased ion transport and macromolecular permeability, and increased adhesion/penetration of total bacteria were observed together with an elevated corticosterone level in the blood [41]. Probiotic treatment by L. rhamnosus R0011 and L. helveticus R0052 ameliorated the MS-induced gut functional abnormalities and bacterial adhesion/penetration, and reduced the corticosterone level. In another rat MS model, depression-like behavior, heightened mRNA level of $\mathrm{CRH}$ in the amygdala, reduced amount of noradrenaline in the brain, and increased peripheral IL-6 were observed; however, administration of B.infantis improved these abnormalities [42]. In another mouse model of depression (exposure to water avoidance stress), pretreatment of a probiotic formulation (B. longum R0175 and L. helveticus R0052) attenuated the HPA axis and ANS activities in response to water avoidance stress and prevented chronic stress-induced decreased hippocampal neurogenesis and expression changes in hypothalamic genes involved in synaptic plasticity. These central effects were associated with restoration of tight junction barrier integrity in stressed mice [43]. In a rat chronic unpredictable mild stress (CUMS) model, Gu et al. [44] reported that L. casei intervention improved the CUMS-induced depressionlike behaviors and the gut microbiota structure changes. Further, L. casei intervention reversed the CUMS-induced expression changes of monoamines dopamine, noradrenaline, and serotonin, and BDNF and its receptor 
TrkB signal molecules. In a chronic social defeat stress model, resilient mice were associated with increased Bifidobacterium counts in fecal samples, compared with susceptible mice, and pretreatment of Bifidobacterium increased the rate of resilient mice to the stress [45].

Regarding the effect of prebiotics, Savignac et al. [46] examined the effects of fructo-oligosaccharides and galacto-oligosaccharides on the expression of BDNF and NMDAR subunits in the rat brain. Prebiotics increased hippocampal BDNF and NR1 subunit expression relative to controls. Galacto-oligosaccharides, in particular, increased plasma peptide YY which increased BDNF expression in SH-SY5Y cell cultures, suggesting a possibility that symbiotics may enrich the gut microbiota, stimulate gut hormone, and increase BDNF expression in the brain. The same research group subsequently demonstrated that feeding neonatal rats by galacto-oligosaccharides increased a NMDAR (GluN2), a synaptic protein (synaptophysin) and BDNF in the hippocampus of 22-day-old rats [47]. This effect remained at day 56 (26 days after the feeding ceased), suggesting the importance of early exposure to prebiotics in the brain development.

All these observations in animal studies indicate that probiotics and prebiotics may have beneficial effects on behavioral and molecular abnormalities, including monoamines, markers for the HPA axis and ANS, inflammation, and BDNF, which have been postulated to play important roles in the pathophysiology of depression.

\section{Evidence of Altered Gut Microbiota in Depression}

Studies on the gut microbiota in MDD are accumulating; however, the results have been diverse from a study to another. Naseribafrouei et al. [48] seem to be the first that reported a comprehensive microbiota profile of fecal samples from MDD patients. They performed an operational taxonomic unit analysis on $16 \mathrm{~S}$ rRNA sequence data in 37 patients with MDD and 18 controls, and reported that the order Bacteroidales, the genus Alistipes, and the genus Oscillibacter were increased, while the family Lachnospiraceae was decreased in their patients compared with the controls. Subsequently, Jiang et al. [49] investigated 46 patients with MDD and 30 healthy controls and found that Bacteroidetes, Proteobacteria, and Actinobacteria were increased, while Firmicutes was decreased in their patients compared with the controls. At the genus level, Enterobacteriaceae and Alistipes were increased and Faecalibacterium was decreased. Of note, Faecalibacterium counts negatively correlated with de- pression severity. In a relatively large number of subjects (58 patients and 63 controls), Zheng et al. [50] reported elevated and reduced numbers of Actinobacteria and Bacteroidetes, respectively. Lin et al. [51] reported that more phylum Firmicutes, less Bacteroidetes, and more genus Prevotella, Klebsiella, Streptococcus, and Clostridium XI were found in MDD patients. The changes of the proportion of Prevotella and Klebsiella were consistent with Hamilton Depression Rating Scale (HAM-D). However, their study was limited by the small sample size (10 patients and 10 controls). Chen et al. [52] examined the microbiota of first-episode drug-naive MDD patients with a special attention to sex difference. They recruited $24 \mathrm{fe}-$ male MDD patients and 24 matched healthy controls and 20 male MDD patients and 20 matched controls. Compared with their healthy counterparts, increased Actinobacteria and decreased Bacteroidetes levels were found in female and male MDD patients, respectively. The most differentially abundant bacterial taxa in female and male MDD patients belonged to phyla Actinobacteria and Bacteroidia, respectively.

Concerning $\alpha$-diversity of the microbiota, Jiang et al. [49] reported that treatment-resistant depressed patients showed higher diversity than healthy controls, which was an unexpected finding because higher $\alpha$-diversity has been considered to be healthy. Other studies reported no significant difference in $\alpha$-diversity between patients and controls $[48,50]$.

The operational taxonomic unit analysis based on next-generation sequencing data has a merit in analyzing comprehensive bacteria species; however, it has a disadvantage in quantification of specific bacteria, especially when the number of the bacteria is small, Lactobacillus species, for example. We then focused our attention to well-known beneficial bacteria, Bifidobacterium and Lactobacillus to count them by using the bacterial rRNA-targeted reverse transcription quantitative polymerase chain reaction method [53] in fecal samples of 43 patients with MDD and 57 healthy controls [54]. The patients had significantly lower Bifidobacterium counts and tended to have lower total Lactobacillus counts than the controls. Frequency of fermented milk consumption was associated with higher Bifidobacterium counts in the patients. In subsequent comparison between patients with bipolar disorder and controls, in contrast, we found no significant difference, although we found a significantly negative correlation between Lactobacillus counts and sleep disturbance score and a significantly negative correlation between Bifidobacterium counts and cortisol levels in the blood of the patients [55].
Kunugi 
In a very large microbiome population cohort (Flemish Gut Flora Project, $N=1,054$ ), Valles-Colomer et al. [56] studied how microbiome features correlate with quality of life and depression. They found that butyrateproducing Faecalibacterium and Coprococcus bacteria were consistently associated with higher quality-of-life indicators. Dialister and Coprococcus spp. were depleted in depression, which was replicated in an independent Dutch sample $(N=1,064)$. They obtained 56 "gut-brain modules" in fecal metagenomes. Among these, they identified the microbial synthesis potential of the dopamine metabolite 3,4-dihydroxyphenylacetic acid as correlating positively with mental quality of life and indicated a potential role of microbial $\gamma$-aminobutyric acid production in depression. 3,4-dihydroxyphenylacetic acid is known to be a predominant biologically active catabolite of quercetin glycosides and to have antioxidant potential of the colonic lumen after the ingestion of the quercetin glycoside-rich onion [57].

The cross-sectional nature of these observational studies precludes inference on the cause-effect relationship. However, 2 studies $[50,58]$ reported that when rats or mice were transplanted with fecal matter from patients with depression, they exhibit depressive and anxiety-like behaviors together with elevated inflammatory status, indicating that altered microbiota in depressed patients has a causal effect.

As described, the findings of microbiota profiles in MDD patients are quite different across the studies. However, there are some consistent points. Multiple studies yielded decreased counts of butyrate-producing bacteria such as Lachnospiraceae and Faecalibacterium [48, 49, 56], and we found decreased counts of Bifidobacterium and Lactobacillus, which also produce short-chain fatty acids (SCFAs) [54]. Further, some studies reported decreased Bacteroidetes [50-52] which is associated with obesity and diabetes $[59,60]$. This may be related to the well-replicated finding of bidirectional association of depression with obesity and diabetes [61].

\section{Clinical Trials of Probiotics}

At least 19 double-blind, randomized, placebo-controlled trials (RCTs) examining the effect of probiotics on depressive symptoms in a variety of disease populations or healthy people have been published $[62,63]$. Goh et al. [62] concluded that the depression-reducing effect of probiotics was significant in those with clinical diagnosis of MDD whereas such an effect was not significant in the general population. However, the evidence is still limited. To my knowledge, there are only 6 RCTs using probiotics in patients with MDD. Notably, 3 of the 6 studies were from Iranian research groups.

The first Iranian study of Akkasheh et al. [64] compared 20 patients with MDD (age 20-55 years) receiving an 8-week intervention with $L$. acidophilus, $L$. casei, and B. bifidum and 20 patients who received placebo. The probiotic supplement group improved in Beck Depression Inventory (BDI) score better than the placebo group $(-5.7 \pm 6.4$ vs. $-1.5 \pm 4.8, p=0.001)$. Moreover, the insulin resistance marker, hs-CRP concentrations, and glutathione concentrations in the blood were improved in the probiotics group better than the placebo group. The second Iranian study [65] examined the add-on effect of 6-week symbiotic supplementation in $40 \mathrm{MDD}$ patients (mean age of 35 years) with moderately ill (17 item HAMD scores of 17-23) who had been treated with fluoxetine $(20 \mathrm{mg} /$ day) for 4 weeks. Symbiotic supplementation included L. acidophilus, L. casei, L. bulgaricus, L. rhamnosus, B. breve, B. longum, and Streptococcus (S.) thermophilus, and $100 \mathrm{mg}$ fructo-oligosaccharide as prebiotic. There was a slightly but significantly greater reduction in the HAM-D score in the symbiotic (+fluoxetine) treated patients (Mean $\pm \mathrm{SD}=-19.25 \pm 1.71)$ than the placebo (+fluoxetine) group (Mean $\pm \mathrm{SD}=-17.75 \pm 2.05$; $p=$ $0.024)$. The third Iranian study [66] compared the effects of probiotic supplementation (L. helveticus R0052 and B. longum R0175; $N=28$ ), prebiotics (galacto-oligosaccharide; $N=27)$, and placebo $(N=26)$ for 8 weeks in patients with MDD who were already treated with antidepressants. Probiotic supplementation resulted in a significant decrease in BDI score $(17.4->9.1)$ compared to the placebo (18.2->15.6) and prebiotic $(19.7->14.1)$ supplementation. There was no significant difference between the prebiotics and placebo groups. Notably, the kynurenine/tryptophan ratio decreased significantly in the probiotic group compared to the placebo group after adjusting for serum isoleucine. In addition, the tryptophan/isoleucine ratio increased significantly in the probiotic group when compared to the placebo group.

Majeed et al. [67] from India recruited 40 MDD patients with comorbid irritable bowel syndrome. Significant improvement in depression severity and irritable bowel syndrome symptoms was observed in the intervention group $(N=20)$ with Bacillus coagulans MTCC after 90 days in comparison with the placebo group $(N=20)$. Serum myeloperoxidase, an inflammatory biomarker, was significantly reduced in the probiotic group in comparison with the placebo group. Rudzki et al. [68] from 
Poland examined the effect of augmentation of SSRI treatment with probiotic bacteria L. Plantarum 299v (LP299v) for 8 weeks on cognitive performance and inflammation-related molecules in MDD patients (mean age 39 years; analyzed for 30 participants in the LP299v group and 30 participants in the placebo group). There was no significant effect of probiotics on the improvement of depressive symptoms assessed with HAM-D. However, the probiotics group showed better improvement in attention task and in episodic verbal learning and memory task, compared to the placebo group. Biochemical monitoring revealed that there was a significant decrease in kynurenine concentration in the LP299v group compared to the placebo group. Reininghaus et al. [69] from Austria reported RCTs on 61 inpatients (mean age of around 30 years) with MDD by probiotics $(N=28)$ or placebo $(N=33)$. The probiotics composed of 9 bacteria species: B. bifidum W23, B. lactis W51, B. lactis W52, $L$. acidophilus W22, L. casei W56, Lacticaseibacillus paracasei W20, L. plantarum W62, L. salivarius W24, and L. lactis W19. Biotin (vitamin B7) was added on for both groups. The microbiota profile became differed after 4 weeks of treatment, that is, Ruminococcus gauvreauii and Coprococcus 3 were more abundant, and $\beta$-diversity was higher in the probiotics group than the placebo group after 28 days. However, improvement of the psychiatric symptoms was not significantly different. Notably, this study group examined the zonulin level in the blood as a marker of intestinal barrier function; however, they did not find any significant effect of time (before $v$. after treatment) or treatment (probiotics v. placebo) on the zonulin level.

There are notable other studies that report the beneficial effects of probiotics ( $L$. casei Shirota) on perceived stress, stress-induced abdominal dysfunction, and sleep disturbances in healthy populations [70,71]. The effect of this probiotic on MDD patients has recently emerged, reporting that better response was associated with abundance of Actinobacteria including Bifidobacterium in the gut microbiota of the patients [72].

\section{Mechanisms}

There are many possible pathways linking the gut microbiota and the pathophysiology of MDD. Those related to the dopamine system, stress response, inflammation, and BDNF are described before. Here, I raise only a few additional factors. Animal studies have revealed that chronic stress induces excessive gut permeability ("Leaky gut") which allows toxic substances such as lipopolysaccharide to act on the intestine or penetrate the body and then leads to inflammation in the gut and eventually to neural inflammation through the toll-like receptor-4 pathway [73]. In line, increased intestinal inflammation induced by lipopolysaccharide or bacterial infection correlates with elevated microglial activation and release of pro-inflammatory cytokines in the brain [74]. Also, such altered gut permeability could be reversed by prebiotic treatment $[75,76]$. It has been demonstrated in humans as well that psychological stress and elevated $\mathrm{CRH}$ increase intestinal permeability $[77,78]$. However, evidence for increased permeability in patients with MDD is still scarce. The study of Calarge et al. [79] might be the only one that examined the relationship between gut permeability and depressive symptoms. They recruited unmedicated adolescent female patients with major depressive episode and healthy subjects to evaluate gut permeability by using the lactulose to mannitol test, the gold standard to evaluate intestinal permeability [80]. They found that lactulose to mannitol ratio was significantly positively associated with depression severity, particularly neurovegetative symptom severity. However, in a clinical trial of the probiotics [69], zonulin level was unrelated to the probiotic treatment. Our recent study also failed to provide supporting evidence for the effect of a probiotic on intestinal permeability assessed with the lactulose to mannitol test [72]. More studies are clearly required to conclude whether gut permeability is elevated in patients with MDD and thus "leaky gut" is substantially involved in the pathogenesis of MDD.

Gut microbiota affects permeability of BBB as well. Braniste et al. [81] reported that GF mice, beginning with intrauterine life, displayed increased BBB permeability during adulthood compared to SPF mice with a normal gut flora. The increased BBB permeability was associated with reduced expression of the tight junction proteins occludin and claudin-5 in the frontal cortex, striatum, and hippocampus. Exposure of GF adult mice to a SPF gut microbiota decreased BBB permeability and up-regulated the expression of the tight junction proteins. Also, monocolonization of the intestine of GF adult mice with either C. tyrobutyricum, a butyrate-producing bacteria, or Bacteroides thetaiotaomicron, which produces mainly acetate and propionate, decreased BBB permeability. SCFAs such as butyrate are known to enhance the integrity of $\mathrm{BBB}$ and the intestinal epithelial barrier by facilitating the assembly of tight junctions [82]. The increased BBB permeability allows pro-inflammatory cytokines and inflammation-inducing proteins such as fibrinogen to penetrate
Kunugi 
the $\mathrm{BBB}$ and enter into the brain, which leads to neural inflammation. This accords with the observation of an elevated CSF-to-serum albumin ratio, an indicator of BBB permeability, in some MDD patients [83, 84]. Further, at least a portion of patients with MDD showed abnormally increased fibrinogen level in their CSF when compared with healthy controls [85].

Another possible mechanism is the reciprocal interaction between the gut microbiota and the ANSs. Dysregulation of the ANSs, that is, overactivation of the sympathetic nervous system and attenuated activity of the parasympathetic nervous system occur under chronic stress. Stress activates the HPA axis and facilitates release of catecholamines such as noradrenaline into the circulatory system which activates the sympathetic nervous system. Noradrenaline influences growth, infectivity and virulence of the enteric bacteria such as the Gram-negative bacteria Yersinia enterocolitica and Escherichia coli in a non-immune manner [86]. Such changes in the gut microbiota may contribute to emotional dysfunctions including depression via activation of immune responses or viscerosensory pathways. On the other hand, beneficial microbes, L. johnsonii, for example, was shown to induce activation of vagal sensory neurons (a main peripheral structure of the parasympathetic nervous system) innervating the gastrointestinal tract [87]. Butyrate is also known to activate intestinal vagus nerves by directly acting on the terminals [88]. The involvement of vagus nerves is supported by the observation that anxiolytic effects of chronic treatment with $L$. rhamnosus (JB-1) were eliminated by vagotomy [89]. In line, vagus nerve stimulation has been shown to be effective in the treatment of MDD [90].

Taken together, SCFA (butyrate, in particular)-producing bacteria could be a next-generation probiotics [91] which could also be a next-generation treatment strategy for MDD. In addition, intervention into lifestyles (healthy diet, exercise, and appropriate use of antibiotics) that influence the gut microbiota $[92,93]$ will be an important strategy in the treatment of MDD.

\section{Conclusion}

Clinical and animal studies have indicated that gut microbiota plays important roles in the central dopamine system, stress response, inflammation, and BDNF, all of which have been suggested to be key molecule/system in the pathophysiology of MDD. The effects on intestinal barrier and BBB permeability and interactions with ANSs

may be involved in the link between gut microbiota and clinical depression. Although the results of microbiota profile of MDD patients varied across studies, there seem to be a consensus that bacteria which produce SCFAs such as butyrate and those protective against metabolic diseases (e.g., Bacteroidetes) might be beneficial. Clinical trials of probiotics on patients with MDD have emerged, and the beneficial effects on depression symptoms and related biological markers have been reported. Therefore, SCFA-producing bacteria could be a next-generation treatment option for clinical depression and intervention into lifestyles that leads to healthier gut microbiota will also be important. Although there is still a long way to achieve adequate understanding the role of the gut-brain axis in MDD, research on this field is promising to elucidate the pathophysiology and new treatment strategies of MDD.

\section{Acknowledgement}

This study was supported by the by the Strategic Research Program for Brain Sciences from Japan Agency for Medical Research and development, AMED, Japan (Grant No. 18dm0107100h0003).

\section{Statement of Ethics}

This is a review study and does not include data collection from human subjects or animal experiments.

\section{Conflict of Interest Statement}

The author received research grants from Yakult Honsha Co., Ltd, Daiichi Sankyo Co., Ltd, and Takeda pharmaceutical Co., Ltd, and Toyobo Co. Ltd, and applied for patents with Toyobo Co. Ltd.

References

Ann Nutr Metab 2021;77(suppl 2):11-20 DOI: $10.1159 / 000518274$
1 Ferrari AJ, Charlson FJ, Norman RE, Patten SB, Freedman G, Murray CJ, et al. Burden of depressive disorders by country, sex, age, and year: findings from the global burden of disease study 2010. PLoS Med. 2013;10(11): e1001547.

2 Rush AJ, Trivedi MH, Wisniewski SR, Stewart JW, Nierenberg AA, Thase ME, et al. Bupropion-SR, sertraline, or venlafaxine-XR after failure of SSRIs for depression. N Engl J Med. 2006;354:1231-42.

3 Kunugi H, Hori H, Adachi N, Numakawa T. Interface between hypothalamic-pituitaryadrenal axis and brain-derived neurotrophic factor in depression. Psychiatry Clin Neurosci. 2010;64(5):447-59. 
4 Kunugi H, Hori H, Ogawa S. Biochemical markers subtyping major depressive disorder. Psychiatry Clin Neurosci. 2015;69(10):597608.

5 Numakawa T, Richards M, Nakajima S, Adachi N, Furuta M, Odaka H, et al. The role of brain-derived neurotrophic factor in comorbid depression: possible linkage with steroid hormones, cytokines, and nutrition. Front Psychiatry. 2014;5:136.

6 Huang TT, Lai JB, Du YL, Xu Y, Ruan LM, Hu $\mathrm{SH}$. Current understanding of gut microbiota in mood disorders: an update of human studies. Front Genet. 2019;10:98.

7 Bastiaanssen TFS, Cussotto S, Claesson MJ, Clarke G, Dinan TG, Cryan JF. Gutted! unraveling the role of the microbiome in major depressive disorder. Harv Rev Psychiatry. 2020;28(1):26-39.

8 Ogawa S, Tsuchimine S, Kunugi H. Cerebrospinal fluid monoamine metabolite concentrations in depressive disorder: a meta-analysis of historic evidence. J Psychiatr Res. 2018; 105:137-46.

9 Ogawa S, Kunugi H. Evidence for reduced homovanillic acid (HVA) in the cerebrospinal fluid of patients with depression. J Affect Disord. 2019;255.

10 Yoon HS, Hattori K, Ogawa S, Sasayama D, Ota M, Teraishi T, et al. Relationships of cerebrospinal fluid monoamine metabolite levels with clinical variables in major depressive disorder. J Clin Psychiatry. 2017 Sep/Oct; 78(8):e947-56.

11 Tanda G, Carboni E, Frau R, Di Chiara G. Increase of extracellular dopamine in the prefrontal cortex: a trait of drugs with antidepressant potential? Psychopharmacology. 1994;115:285-8.

12 Hori $\mathrm{H}$, Kunugi $\mathrm{H}$. The efficacy of pramipexole, a dopamine receptor agonist, as an adjunctive treatment in treatment-resistant depression: an open-label trial. ScientificWorldJournal. 2012;2012:372474.

13 Hori H, Kunugi H. Dopamine agonist-responsive depression. Psychogeriatrics. 2013; 13(3):189-95

14 Chiba S, Numakawa T, Ninomiya M, Yoon HS, Kunugi H. Cabergoline, a dopamine receptor agonist, has an antidepressant-like property and enhances brain-derived neurotrophic factor signaling. Psychopharmacology. 2010;211(3):291-301.

15 Adachi N, Yoshimura A, Chiba S, Ogawa S, Kunugi $\mathrm{H}$. Rotigotine, a dopamine receptor agonist, increased BDNF protein levels in the rat cortex and hippocampus. Neurosci Lett. 2018;662:44-50.

16 Holsboer F. The corticosteroid receptor hypothesis of depression. Neuropsychopharmacology. 2000;23:477-501.

17 Kunugi H, Urushibara T, Nanko S. Combined DEX/CRH test among Japanese patients with major depression. J Psychiatr Res. 2004 MarApr;38(2):123-8.
18 Kunugi H, Ida I, Owashi T, Kimura M, Inoue Y, Nakagawa S, et al. Assessment of the dexamethasone/CRH test as a state-dependent marker for hypothalamic-pituitary-adrenal (HPA) axis abnormalities in major depressive episode: a Multicenter Study. Neuropsychopharmacology. 2006;31(1):212-20.

19 Carpenter LL, Ross NS, Tyrka AR, Anderson GM, Kelly M, Price LH. Dex/CRH test cortisol response in outpatients with major depression and matched healthy controls. Psychoneuroendocrinology. 2009;34:1208-13.

20 Wahlberg K, Ghatan PH, Modell S, Nygren A, Ingvar M, Asberg M, et al. Suppressed neuroendocrine stress response in depressed women on job-stress-related long-term sick leave: a stable marker potentially suggestive of preexisting vulnerability. Biol Psychiatry. 2009; 65(9):742-7.

21 Yehuda R, Southwick SM, Krystal JH, Bremner D, Charney DS, Mason JW. Enhanced suppression of cortisol following dexamethasone administration in posttraumatic stress disorder. Am J Psychiatry. 1993;150:83-6.

22 Gaab J, Hüster D, Peisen R, Engert V, Schad $\mathrm{T}$, Schürmeyer TH, et al. Low-dose dexamethasone suppression test in chronic fatigue syndrome and health. Psychosom Med. 2002; 64:311-8.

23 Wingenfeld $\mathrm{K}$, Wagner $\mathrm{D}$, Schmidt $\mathrm{I}$, Meinlschmidt G, Hellhammer DH, Heim C. The low-dose dexamethasone suppression test in fibromyalgia. J Psychosom Res. 2007; 62:85-91.

24 Koch C, Wilhelm M, Salzmann S, Rief W, Euteneuer F. A meta-analysis of heart rate variability in major depression. Psychol Med. 2019;49(12):1948-57.

25 Zhang X, Qing MJ, Rao YH, Guo YM. Adjunctive vagus nerve stimulation for treatment-resistant depression: a quantitative analysis. Psychiatr Q. 2020;91(3):669-79.

26 Miller AH, Maletic V, Raison CL. Inflammation and its discontents: the role of cytokines in the pathophysiology of major depression. Biol Psychiatry. 2009;65:732-41.

27 Sasayama D, Hattori K, Wakabayashi C, Teraishi $\mathrm{T}$, Hori $\mathrm{H}$, Ota $\mathrm{M}$, et al. Increased cerebrospinal fluid interleukin-6 levels in patients with schizophrenia and those with major depressive disorder. J Psychiatr Res. 2013;47(3):401-6.

28 Wang AK, Miller BJ. Meta-analysis of cerebrospinal fluid cytokine and tryptophan catabolite alterations in psychiatric patients: comparisons between schizophrenia, bipolar disorder, and depression. Schizophr Bull. 2018;44(1):75-83.

29 Dantzer R, O'Connor JC, Lawson MA, Kelley KW. Inflammation-associated depression: from serotonin to kynurenine. Psychoneuroendocrinology. 2011;36:426-36.

30 Teraishi T, Hori H, Sasayama D, Matsuo J, Ogawa S, Ota M, et al. (13)C-tryptophan breath test detects increased catabolic turnover of tryptophan along the kynurenine pathway in patients with major depressive disorder. Sci Rep. 2015;5:15994.
31 Ogawa S, Fujii T, Koga N, Hori H, Teraishi T, Hattori K, et al. Plasma L-tryptophan concentration in major depressive disorder: new data and meta-analysis. J Clin Psychiatry. 2014; 75(9):e906-15.

32 Ogawa S, Koga N, Hattori K, Matsuo J, Ota M, Hori $\mathrm{H}$, et al. Plasma amino acid profile in major depressive disorder: analyses in two independent case-control sample sets. J Psychiatr Res. 2018;96:23-32.

33 Duman RS, Monteggia LM. A neurotrophic model for stress-related mood disorders. Biol Psychiatry. 2006;59(12):1116-27.

34 Chiba S, Numakawa T, Ninomiya M, Richards MC, Wakabayashi C, Kunugi H. Chronic restraint stress causes anxiety- and depression-like behaviors, downregulates glucocorticoid receptor expression, and attenuates glutamate release induced by brain-derived neurotrophic factor in the prefrontal cortex. Prog Neuropsychopharmacol Biol Psychiatry. 2012;39(1):112-9.

35 Numakawa T, Kumamaru E, Adachi N, Yagasaki Y, Izumi A, Kunugi H. Glucocorticoid receptor interaction with $\operatorname{TrkB}$ promotes BDNF-triggered PLC-gamma signaling for glutamate release via a glutamate transporter. Proc Natl Acad Sci U S A. 2009;106(2):64752.

36 Numakawa T, Adachi N, Richards M, Chiba $\mathrm{S}$, Kunugi $\mathrm{H}$. Brain-derived neurotrophic factor and glucocorticoids: reciprocal influence on the central nervous system. Neuroscience. 2013;239:157-72

37 Mizui T, Hattori K, Ishiwata S, Hidese S, Yoshida S, Kunugi H, et al. Cerebrospinal fluid BDNF pro-peptide levels in major depressive disorder and schizophrenia. I Psychiatr Res. 2019;113:190-8.

38 Sudo N, Chida Y, Aiba Y, Sonoda J, Oyama N, $\mathrm{Yu} \mathrm{XN}$, et al. Postnatal microbial colonization programs the hypothalamic-pituitary-adrenal system for stress response in mice. J Physiol. 2004;558(Pt 1):263-75.

39 Crumeyrolle-Arias M, Jaglin M, Bruneau A, Vancassel S, Cardona A, Daugé V, et al. Absence of the gut microbiota enhances anxietylike behavior and neuroendocrine response to acute stress in rats. Psychoneuroendocrinology. 2014;42:207-17.

40 Liu WH, Chuang HL, Huang YT, Wu CC, Chou GT, Wang S, et al. Alteration of behavior and monoamine levels attributable to Lactobacillus plantarum PS128 in germ-free mice. Behav Brain Res. 2016;298:202-9.

41 Gareau MG, Jury J, MacQueen G, Sherman $\mathrm{PM}$, Perdue MH. Probiotic treatment of rat pups normalises corticosterone release and ameliorates colonic dysfunction induced by maternal separation. Gut. 2007;56(11):15228.

42 Desbonnet L, Garrett L, Clarke G, Kiely B, Cryan JF, Dinan TG. Effects of the probiotic Bifidobacterium infantis in the maternal separation model of depression. Neuroscience. 2010;170(4):1179-88. 
43 Ait-Belgnaoui A, Colom A, Braniste V, Ramalho L, Marrot A, Cartier C, et al. Probiotic gut effect prevents the chronic psychological stress-induced brain activity abnormality in mice. Neurogastroenterol Motil. 2014;26(4): 510-20.

44 Gu F, Wu Y, Liu Y, Dou M, Jiang Y, Liang H. Lactobacillus casei improves depression-like behavior in chronic unpredictable mild stress-induced rats by the BDNF-TrkB signal pathway and the intestinal microbiota. Food Funct. 2020;11(7):6148-57.

45 Yang C, Fujita Y, Ren Q, Ma M, Dong C, Hashimoto K. Bifidobacterium in the gut microbiota confer resilience to chronic social defeat stress in mice. Sci Rep. 2017;7:45942.

46 Savignac HM, Corona G, Mills H, Chen L, Spencer JP, Tzortzis G, et al. Prebiotic feeding elevates central brain derived neurotrophic factor, N-methyl-D-aspartate receptor subunits and D-serine. Neurochem Int. 2013; 63(8):756-64.

47 Williams S, Chen L, Savignac HM, Tzortzis G, Anthony DC, Burnet PW. Neonatal prebiotic (BGOS) supplementation increases the levels of synaptophysin, GluN2A-subunits and $\mathrm{BDNF}$ proteins in the adult rat hippocampus. Synapse. 2016;70(3):121-4.

48 Naseribafrouei A, Hestad K, Avershina E, Sekelja M, Linløkken A, Wilson R, et al. Correlation between the human fecal microbiota and depression. Neurogastroenterol Motil. 2014;26(8):1155-62.

49 Jiang $\mathrm{H}$, Ling Z, Zhang $\mathrm{Y}$, Mao H, Ma Z, Yin $Y$, et al. Altered fecal microbiota composition in patients with major depressive disorder. Brain Behav Immun. 2015;48:186-94.

50 Zheng P, Zeng B, Zhou C, Liu M, Fang Z, Xu $\mathrm{X}$, et al. Gut microbiome remodeling induces depressive-like behaviors through a pathway mediated by the host's metabolism. Mol Psychiatry. 2016;21(6):786-96.

51 Lin P, Ding B, Feng C, Yin S, Zhang T, Qi X, et al. Prevotella and Klebsiella proportions in fecal microbial communities are potential characteristic parameters for patients with major depressive disorder. J Affect Disord. 2017;207:300-4.

52 Chen JJ, Zheng P, Liu YY, Zhong XG, Wang HY, Guo YJ, et al. Sex differences in gut microbiota in patients with major depressive disorder. Neuropsychiatr Dis Treat. 2018;14: 647-55.

53 Matsuda K, Tsuji H, Asahara T, Matsumoto K, Takada T, Nomoto K. Establishment of an analytical system for the human fecal microbiota, based on reverse transcription-quantitative PCR targeting of multicopy rRNA molecules. Appl Environ Microbiol. 2009;75: 1961-9.

54 Aizawa E, Tsuji H, Asahara T, Takahashi T, Teraishi T, Yoshida S, et al. Possible association of Bifidobacterium and Lactobacillus in the gut microbiota of patients with major depressive disorder. J Affect Disord. 2016;202: 254-7.
55 Aizawa E, Tsuji H, Asahara T, Takahashi T, Teraishi T, Yoshida S, et al. Bifidobacterium and Lactobacillus counts in the gut microbiota of patients with bipolar disorder and healthy controls. Front Psychiatry. 2019;9: 730.

56 Valles-Colomer M, Falony G, Darzi Y, Tigchelaar EF, Wang J, Tito RY, et al. The neuroactive potential of the human gut microbiota in quality of life and depression. Nat Microbiol. 2019;4(4):623-32.

57 Tang Y, Nakashima S, Saiki S, Myoi Y, Abe N, Kuwazuru S, et al. 3,4-Dihydroxyphenylacetic acid is a predominant biologically-active catabolite of quercetin glycosides. Food Res Int. 2016;89(Pt 1):716-23.

58 Kelly JR, Borre Y, O’ Brien C, Patterson E, El Aidy S, Deane J, et al. Transferring the blues: depression-associated gut microbiota induces neurobehavioural changes in the rat. J Psychiatr Res. 2016;82:109-18.

59 Ley RE, Turnbaugh PJ, Klein S, Gordon JI. Microbial ecology: human gut microbes associated with obesity. Nature. 2006;444(7122): 1022-3.

60 Elena RM, Gabriela GD, Arnulfo GC, Enrique CA. Studying the gut microbiome of Latin America and Hispanic/Latino Populations. Insight into obesity and diabetes: systematic review. Curr Diabetes Rev. 2019;15(4):294301.

61 Luppino FS, de Wit LM, Bouvy PF, Stijnen T, Cuijpers P, Penninx BW, et al. Overweight, obesity, and depression: a systematic review and meta-analysis of longitudinal studies. Arch Gen Psychiatry. 2010;67(3):220-9.

62 Goh KK, Liu YW, Kuo PH, Chung YE, Lu ML, Chen $\mathrm{CH}$. Effect of probiotics on depressive symptoms: a meta-analysis of human studies. Psychiatry Res. 2019;282:112568.

63 Mörkl S, Butler MI, Holl A, Cryan JF, Dinan TG. Probiotics and the microbiota-gut-brain axis: focus on psychiatry. Curr Nutr Rep. 2020;9(3):171-82.

64 Akkasheh G, Kashani-Poor Z, Tajabadi-Ebrahimi M, Jafari P, Akbari H, Taghizadeh M, et al. Clinical and metabolic response to probiotic administration in patients with major depressive disorder: a randomized, doubleblind, placebo-controlled trial. Nutrition. 2016;32(3):315-20.

65 Ghorbani Z, Nazari S, Etesam F, Nourimajd S, Ahmadpanah M, Razeghi Jahromi S. The effect of synbiotic as an adjuvant therapy to fluoxetine in moderate depression: a randomized multicenter trial. Arch Neurosci. 2018; 5(2):e60507.

66 Kazemi A, Noorbala AA, Azam K, Eskandari $\mathrm{MH}$, Djafarian K. Effect of probiotic and prebiotic vs placebo on psychological outcomes in patients with major depressive disorder: a randomized clinical trial. Clin Nutr. 2019; 38(2):522-8.
67 Majeed M, Nagabhushanam K, Arumugam S, Majeed S, Ali F. Bacillus coagulans MTCC 5856 for the management of major depression with irritable bowel syndrome: a randomised, double-blind, placebo controlled, multi-centre, pilot clinical study. Food Nutr Res. 2018; 62.

68 Rudzki L, Ostrowska L, Pawlak D, Małus A, Pawlak K, Waszkiewicz N, et al. Probiotic Lactobacillus Plantarum 299v decreases kynurenine concentration and improves cognitive functions in patients with major depression: a double-blind, randomized, placebo controlled study. Psychoneuroendocrinology. 2019;100:213-22.

69 Reininghaus EZ, Platzer M, KohlhammerDohr A, Hamm C, Mörkl S, Bengesser SA, et al. PROVIT: supplementary probiotic treatment and vitamin B7 in depression-A randomized controlled trial. Nutrients. 2020; 12(11):3422.

70 Kato-Kataoka A, Nishida K, Takada M, Kawai M, Kikuchi-Hayakawa H, Suda K, et al. Fermented milk containing Lactobacillus casei strain Shirota preserves the diversity of the gut microbiota and relieves abdominal dysfunction in healthy medical students exposed to academic stress. Appl Environ Microbiol. 2016;82(12):3649-58.

71 Takada M, Nishida K, Gondo Y, KikuchiHayakawa H, Ishikawa H, Suda K, et al. Beneficial effects of Lactobacillus casei strain Shirota on academic stress-induced sleep disturbance in healthy adults: a double-blind, randomised, placebo-controlled trial. Benef Microbes. 2017;8(2):153-62.

72 Otaka M, Kikuchi-Hayakawa H, Ogura J, Ishikawa H, Yomogida Y, Ota M, et al. Effect of Lacticaseibacillus paracasei strain Shirota on improvement in depressive symptoms, and its association with abundance of Actinobacteria in Gut Microbiota. Microorganisms. 2021;9(5):1026.

73 Gárate I, Garcia-Bueno B, Madrigal JL, Caso JR, Alou L, Gomez-Lus ML, et al. Stress-induced neuroinflammation: role of the Tolllike receptor-4 pathway. Biol Psychiatry. 2013;73(1):32-43.

74 Riazi K, Galic MA, Kuzmiski JB, Ho W, Sharkey KA, Pittman QJ. Microglial activation and TNFalpha production mediate altered CNS excitability following peripheral inflammation. Proc Natl Acad Sci U S A. 2008; 105: 17151-6.

75 Donato KA, Gareau MG, Wang YJJ, Sherman PM. Lactobacillus rhamnosus GG attenuates interferon-\{gamma\} and tumour necrosis factor-alpha-induced barrier dysfunction and pro-inflammatory signalling. Microbiology. 2010;156(Pt 11):3288-97.

76 Zareie M, Johnson-Henry K, Jury J, Yang PC, Ngan BY, McKay DM, et al. Probiotics prevent bacterial translocation and improve intestinal barrier function in rats following chronic psychological stress. Gut. 2006; 55(11):1553-60. 
77 Vanuytsel T, van Wanrooy S, Vanheel H, Vanormelingen C, Verschueren S, Houben E, et al. Psychological stress and corticotropinreleasing hormone increase intestinal permeability in humans by a mast cell-dependent mechanism. Gut. 2014;63(8):1293-9.

78 Velin AK, Ericson AC, Braaf Y, Wallon C, Söderholm JD. Increased antigen and bacterial uptake in follicle associated epithelium induced by chronic psychological stress in rats. Gut. 2004;53(4):494-500.

79 Calarge CA, Devaraj S, Shulman RJ. Gut permeability and depressive symptom severity in unmedicated adolescents. J Affect Disord. 2019;246:586-94.

80 Camilleri M, Nadeau A, Lamsam J, Nord SL, Ryks M, Burton D, et al. Understanding measurements of intestinal permeability in healthy humans with urine lactulose and mannitol excretion. Neurogastroenterol Motil. 2010;22:e15-26.

81 Braniste V, Al-Asmakh M, Kowal C, Anuar F, Abbaspour A, Tóth M, et al. The gut microbiota influences blood-brain barrier permeability in mice. Sci Transl Med. 2014;6(263): 263ra158.

82 Peng L, He Z, Chen W, Holzman IR, Lin J. Effects of butyrate on intestinal barrier function in a Caco- 2 cell monolayer model of intestinal barrier. Pediatr Res. 2007;61:37-41.
83 Gudmundsson P, Skoog I, Waern M, Blennow K, Pálsson S, Rosengren L, et al. The relationship between cerebrospinal fluid biomarkers and depression in elderly women. Am J Geriatr Psychiatry. 2007;15:832.

84 Bechter K, Reiber H, Herzog S, Fuchs D, Tumani H, Maxeiner HG. Cerebrospinal fluid analysis in affective and schizophrenic spectrum disorders: identification of subgroups with immune responses and blood-CSF barrier dysfunction. J Psychiatr Res. 2010;44: 321-30.

85 Hattori K, Ota M, Sasayama D, Yoshida S, Matsumura R, Miyakawa T, et al. Increased cerebrospinal fluid fibrinogen in major depressive disorder. Sci Rep. 2015;5:11412.

86 Lyte M, Vulchanova L, Brown DR. Stress at the intestinal surface: catecholamines and mucosa-bacteria interactions. Cell Tissue Res. 2011;343(1):23-32.

87 Tanida M, Yamano T, Maeda K, Okumura N, Fukushima Y, Nagai K. Effects of intraduodenal injection of Lactobacillus johnsonii La1 on renal sympathetic nerve activity and blood pressure in urethane-anesthetized rats. Neurosci Lett. 2005;389:109-14.
88 Lal S, Kirkup AJ, Brunsden AM, Thompson DG, Grundy D. Vagal afferent responses to fatty acids of different chain length in the rat. Am J Physiol Gastrointest Liver Physiol. 2001; 281:G907-15.

89 Bravo JA, Forsythe P, Chew MV, Escaravage E, Savignac HM, Dinan TG, et al. Ingestion of Lactobacillus strain regulates emotional behavior and central GABA receptor expression in a mouse via the vagus nerve. Proc Nat Acad Sci U S A. 2011;108:16050-5.

90 Carreno FR, Frazer A. Vagal nerve stimulation for treatment-resistant depression. Neurotherapeutics. 2017 Jul;14(3):716-27.

91 Boesmans L, Valles-Colomer M, Wang J, Eeckhaut V, Falony G, Ducatelle R, et al. Butyrate producers as potential next-generation probiotics: safety assessment of the administration of Butyricicoccus pullicaecorum to healthy volunteers. mSystems. 2018;3(6):e00094-18.

92 Dash S, Clarke G, Berk M, Jacka FN. The gut microbiome and diet in psychiatry: focus on depression. Curr Opin Psychiatry. 2015; 28(1):1-6.

93 Juárez-Fernández M, Porras D, García-Mediavilla MV, Román-Sagüillo S, González-Gallego J, Nistal E, et al. Aging, gut microbiota and metabolic diseases: management through physical exercise and nutritional interventions. Nutrients. 2020;13(1):16. 